\title{
DIVERSIFICACIÓN DE LA ACTIVIDAD TURÍSTICA DE BALNEARIOS: EL CASO DE PEHUÉN CO, ARGENTINA
}

\author{
María Luján Bustos ${ }^{1,2}$ \\ Federico Ferrelli ${ }^{1,2}$ \\ María Cintia Piccolo ${ }^{1,2}$
}

\section{RESUMEN}

Para desarrollar un Manejo Integral Costero de localidades turísticas es necesario identificar posibles alternativas que ayuden a diversificar su economía. La localidad de Pehuén Co (provincia de Buenos Aires, Argentina) es un típico caso de turismo sin diversificación. Se realizó un análisis de su oferta y demanda turística mediante encuestas y relevamientos. Esto permitió trazar una zonificación del área de estudio según el grado de desarrollo turístico. Como resultado se identifican nuevas zonas con potencial turístico que permitiría la diversificación de la economía local y regional. Estas alternativas potenciarían un uso sustentable de los recursos y atractivos de Pehuén Co.

Palabras clave: Turismo de balnearios; diversificación; Pehuén Co; Argentina.

\section{Diversification of touristic activities in coastal towns: the case of Pehuén Co, Argentina}

\section{ABSTRACT}

The knowledge about touristic diversification in coastal towns is necessary to develop an Integrated Coastal Zone Management. Pehuen Co city (Buenos Aires, Argentina) is a

Recibido: 17 de marzo de 2015

Devuelto para su revisión: 3 de julio de 2015

Aceptado: 4 de noviembre de 2015

1 Departamento de Geografía y Turismo. Universidad Nacional del Sur. C/ 12 de Octubre y San Juan, $4^{\circ}$ piso. (8000) BAHÍA BLANCA. Provincia de Buenos Aires (Argentina).

2 Instituto Argentino de Oceanografía. CONICET. Camino La Carrindanga, km 7,5. (8000) BAHÍA BLANCA. Provincia de Buenos Aires (Argentina).

E-mail: lujan.bustos@uns.edu.ar,fferrelli@criba.edu.ar,ofpiccol@criba.edu.ar 
typical case to study the diversification of touristic activities. We analyzed tourism supply and demand with surveys. This was useful to delimit regions, depending on the degree of tourism development. New regions with touristic potential were identified. These regions could be useful to generate local and regional economic diversification. The study provides alternatives that would enhance a sustainable use of resources in Pehuen Co.

Keywords: Coastal town tourism; diversification; Pehuen Co; Argentina.

\section{INTRODUCCIÓN}

El turismo se ha convertido en los últimos años en una de las actividades más dinámicas de las economías actuales debido, entre otros factores, a los cambios del consumidor turístico (Molina Collado et al., 2007). El turismo se define como las actividades que realizan las personas durante sus viajes y estancias en lugares distintos al de su entorno habitual, por un período de tiempo mínimo de una noche e inferior a un año, con fines de ocio, por negocios y otros (OMT, 1998).

Las ciudades costeras son las que más visitas turísticas reciben en el Mundo (OMT, 2009). La decisión de elegir el destino principal al momento de realizar un viaje de turismo es fundamental para las personas interesadas en disfrutar un viaje de placer (OMT, 2008). Diversos aspectos motivan la toma de esta decisión, entre ellos se destacan los atractivos que posea el lugar, como por ejemplo el clima (Gómez Martín, 1999; Scott y McBoyle, 2001; Cengiz et al., 2008).

Superpuesto a las complejas interrelaciones entre los factores naturales que intervienen en la costa, existe una marcada influencia antrópica. Si bien la población mundial ha centrado su desarrollo y evolución a partir de asentamientos costeros, es solo en la última mitad del siglo XX en que se ha producido una verdadera invasión de la costa. El cambio en las costumbres y la necesidad de emplear el tiempo libre debido a la modernización industrial y las modificaciones en las condiciones laborales dio lugar al desarrollo de la industria del turismo. Ello planteó un rápido crecimiento de las localidades costeras y la construcción de nuevos centros urbanos, pero muy raramente con un esquema urbanístico que preservara el ambiente natural (Perillo, 1992).

En los países desarrollados rápidamente se observaron las consecuencias de asentamientos urbanos no planificados. De inmediato se organizaron estudios de las costas y se desarrollaron programas de manejo y ordenamiento costero. Por ejemplo, para examinar los problemas de contaminación marina en un contexto interdisciplinario se creó en 1969 el Grupo Mixto de Expertos sobre los Aspectos Científicos de la Protección del Medio Marino (GESAMP) (COI, 2004).

A partir del mandato de la Cumbre de la Tierra de Río en 1992 el Manejo Costero Integrado (MIC) ganó terreno entre las estrategias de gestión en las distintas regiones del mundo. La importancia del mismo reside en que constituye un proceso que une gobierno y comunidades, ciencia y manejo, intereses públicos y privados en la elaboración e implementación de un plan integrado de conservación y desarrollo de los ecosistemas y recursos costeros (Moreno Castillo, 2007). El propósito del MIC es mejorar la calidad 
de vida de las comunidades que dependen de los recursos costeros, así como mantener la productividad y la biodiversidad de esos ecosistemas en el tiempo (GESAMP, 1999).

El MIC es un proceso continuo y dinámico que guía el uso, el desarrollo sustentable y la protección de áreas costeras (Leslie y McLeod, 2007; Chang et al., 2008; Douvere, 2008). Para una base real del MIC es necesario el estudio del clima, la geomorfología, la urbanización y la economía, la cual, para este tipo de ambientes, suele basarse en el turismo o la actividad portuaria (Comisión Europea, 2001).

En ese sentido, Argentina carece de este tipo de iniciativas desde un punto de vista integrado. La transformación en el uso de la tierra, junto con la expansión de la infraestructura costera incrementó el deterioro de estos hábitats. A esto debe sumarse que la legislación argentina es escasa respecto al tratamiento de las zonas costeras (Dadon et $a l .$, 2002). La falta de planificación en la expansión urbana y de infraestructura ocasiona graves inconvenientes a los residentes y al gobierno local. Ante el retroceso de las costas las autoridades deben ocuparse de las exigencias de los propietarios de viviendas cercanas a la playa que ven el avance del mar sobre sus tierras.

El surgimiento de balnearios en la costa de la provincia de Buenos Aires y en el Sur de Argentina respondió a un modelo de desarrollo espontáneo, sin planes previos y con una acción secundaria del Estado (Del Pozo, 2001). Una de las modalidades más observadas fue la iniciativa de los propietarios de campos costeros en lotear sus tierras y venderlas en subasta pública. Esta falta de criterio conservador en la creación y urbanización de los balnearios, sumado al rápido incremento de la demanda de tierras para el turismo representa un gran peligro para las costas (Wong, 1998; Suárez de Vivero y Rodríguez Mateos, 2005). No obstante, en el Sur del país y, especialmente, en el Sur de la provincia de Buenos Aires, las pequeñas localidades costeras continúan en expansión. En su mayoría lo hacen sin ningún tipo de plan de manejo y basando su economía principalmente en el turismo de balnearios. Por ello, es necesario encontrar alternativas a este tipo de turismo. Esta actividad se concentra solo en los meses característicos de verano y fines de semana de otoño o primavera. En invierno o días de lluvia los balnearios permanecen inactivos sin posibilidades de generar ganancias económicas. Este es el caso de la villa balnearia de Pehuén Co, localidad que se encuentra en el Sudoeste de

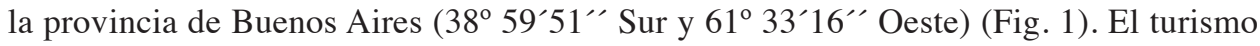
de sol y playa representa la actividad económica más importante de esta villa. Se han realizado trabajos de investigación sobre diferentes temas del turismo en la localidad. Se analizó el potencial turístico de su oferta y demanda (Cutidiano, 2010; Bustos y Piccolo, 2012) y la incidencia que tuvo la repavimentación de la ruta de acceso a la villa para su crecimiento turístico (Sánchez, 2010). Además, se propusieron ofertas de turismo complementarias al atractivo principal (playa), como el turismo ecológico (Bartelucci, 1994; Del Pozo et al., 2000), cultural (Ausili, 2005) y científico (Giulietti, 2010; D’Amato, 2011). Sin embargo, estos estudios se han realizado en forma independiente y no integrada. El MIC es necesario para obtener una economía sustentable en el tiempo y que proteja los recursos naturales. Por ello, son necesarios estudios iniciales para conocer qué áreas son factibles de desarrollarse económicamente. Por este motivo, el objetivo del presente trabajo es analizar las características básicas de la actividad turística en la 
localidad de Pehuén Co (provincia de Buenos Aires, Argentina) con el fin de encontrar potenciales áreas y actividades que permitan diversificar el turismo y elaborar un MIC.

\section{Figura 1 \\ LOCALIZACIÓN DEL BALNEARIO PEHUÉN CO EN EL SUDOESTE DE LA PROVINCIA DE BUENOS AIRES}
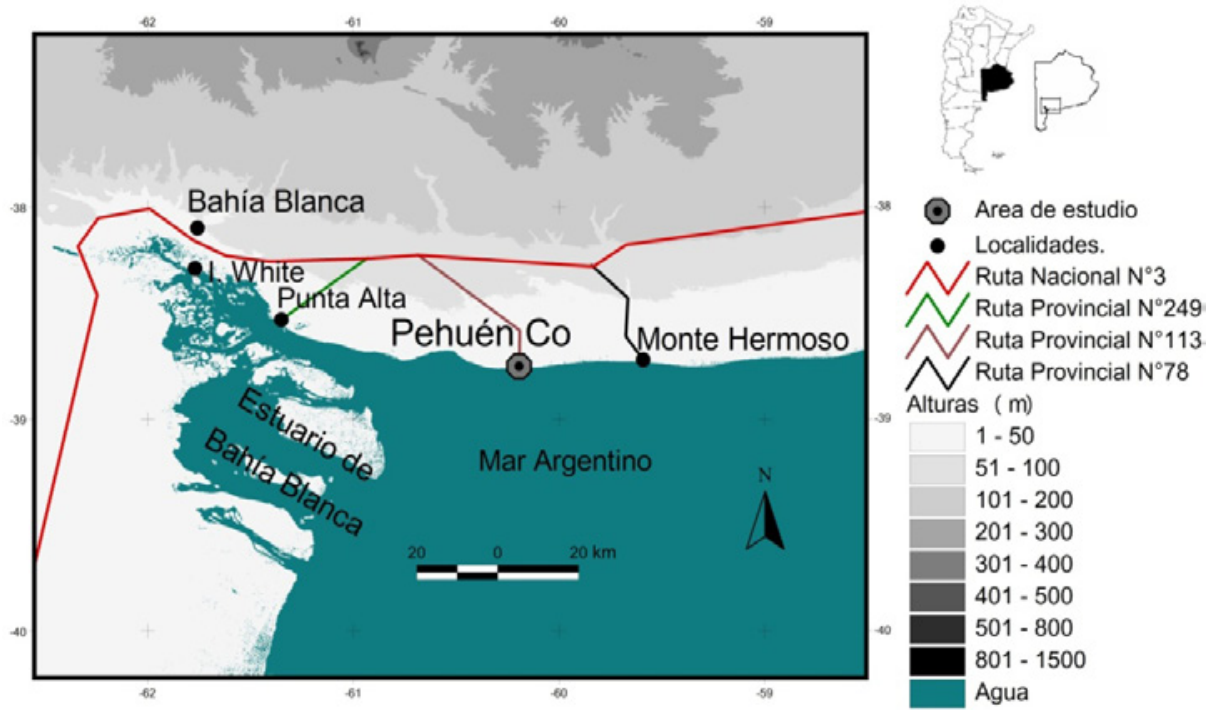

Fuente: elaboración propia.

\section{1. Área de estudio}

Pehuén Co es una localidad costera que se encuentra sobre una saliente conocida como punta Pehuén Co, en la cual está emplazado el centro urbano. Esta característica le otorga rasgos especiales en cuanto a su dinámica y morfología costera. En general, su costa es baja y arenosa con un ancho promedio de playa de $143 \mathrm{~m}$, enmarcada por una línea de médanos frontales. Los mismos forman parte de un complejo de médanos de aproximadamente $6 \mathrm{~km}$ de ancho medio. En los sectores más alejados de la costa los médanos son de menor altura y se encuentran cubiertos de vegetación. En las proximidades de la costa alcanzan una altura de $10 \mathrm{~m}$ aproximadamente con médanos sin vegetar, médanos cubiertos por tamariscos (Tamarix gallica), acantilados artificiales y afloramientos de arena cementada (Calo et al., 1998; Bustos, 2012).

El desarrollo de la villa es paralela a la costa la cual tiene una orientación Este - Oeste. No posee edificaciones en altura superiores a tres pisos y en todas las viviendas se observa un parque o jardín alrededor de la residencia. Conserva todas sus calles sin asfalto sobre un plano irregular. La mayor parte de las calles se encuentran muy forestadas con diferentes especies arbóreas, predominando pinos y eucaliptos. No posee zonas de actividad 
industrial y el área comercial es muy reducida y arbolada (Bustos, 2012). La villa es un núcleo urbano, netamente balneario, que fue fundado en 1948 y a la fecha posee 900 habitantes permanentes (Delegación Municipal de Pehuén Co, 2012). Durante la época estival visitan Pehuén Co más de 600.000 visitantes (Dirección de Turismo de Coronel Rosales, 2012). De diciembre a marzo se genera la mayor actividad turística llegando a superar los 20.000 visitantes diarios.

El área de estudio se encuentra dentro de los climas templados con una temperatura media anual de $14,3{ }^{\circ} \mathrm{C}$. Los veranos e inviernos están bien diferenciados térmicamente. Los meses más fríos tiene una temperatura media de $10{ }^{\circ} \mathrm{C}$ (abril a agosto). El verano es caluroso y la temperatura media para estos meses es de $19{ }^{\circ} \mathrm{C}$ (septiembre a marzo). Es una región de períodos húmedos y secos alternativos. Normalmente las lluvias se concentran en primavera-verano y en otoño, siendo mínimas en invierno (Bustos et al., 2011).

El estudio de los índices medios y extremos de confort para el período 2005-2009, dio por resultado rangos de peligro para las actividades al aire libre de los habitantes en verano e invierno (Bustos y Piccolo, 2011). En el invierno los días con temperaturas mínimas muy bajas (menores a $0{ }^{\circ} \mathrm{C}$ ) generaron horas con sensaciones térmicas de peligro por enfriamiento y valores que podrían afectar la resistencia de una persona inactiva. Julio es el mes invernal más apropiado para actividades al aire libre debido a que sus temperaturas son más moderadas. En verano, diciembre es el mes óptimo para realizar actividades turísticas. En el resto de los meses de verano los períodos más riesgosos para una ola de calor se presentaron desde el mediodía hasta las últimas horas de la tarde. Los días más calurosos que evidencian desconfort coinciden con el predominio del viento del sector Norte (Bustos y Piccolo, 2011).

\section{METODOLOGÍA}

Los recursos turísticos son los que generan la voluntad de las personas de movilizarse hacia un determinado lugar, es por ello que son la materia prima del turismo (Montaner Montejano, 1996). Para determinar los atractivos turísticos de Pehuén Co y su área de influencia se realizó un relevamiento visual y se analizó la información brindada por la Dirección de Turismo de la Municipalidad de Coronel Rosales (DTCR). Con estos datos se efectuó una descripción de cada atractivo y se realizaron los mapas turísticos de Pehuén Co y su zona limítrofe.

Con el fin de determinar las características y preferencias de la demanda turística de Pehuén Co se realizaron encuestas. En estas encuestas no se discriminó entre turistas (personas que permanecen en el lugar mínimo una noche) y recreacionistas (personas que no pernoctan en el lugar), sino que se consideró a ambos como visitantes. Se usaron los datos de las encuestas de la DTCR registrados entre las temporadas 2005/06 a 2010/11 y encuestas de elaboración propia realizadas en la temporada 2010/11. Tanto las encuestas de la DTCR como las de elaboración propia se realizaron en los meses de enero y febrero. En la tabla I se presenta el número de encuestas por temporada.

En las encuestas las preguntas fueron abiertas y cerradas según la metodología presentada por Améringo (1993). Esta técnica permitió evaluar la composición de la demanda turística de la localidad balnearia con el objetivo de cualificar al visitante. Para aplicar- 
Tabla 1

CANTIDAD DE ENCUESTAS REALIZADAS POR AÑO

\begin{tabular}{|c|c|}
\hline Temporada & Cantidad de encuestas \\
\hline $2005 / 06$ & 1600 \\
\hline $2006 / 07$ & 1800 \\
\hline $2007 / 08$ & 1815 \\
\hline $2008 / 09$ & 908 \\
\hline $2009 / 10$ & 800 \\
\hline $2010 / 11$ & 800 \\
\hline $2010 / 11$ (elaboración propia) & 200 \\
\hline
\end{tabular}

Fuente: Modificado de la DTCR.

las, se utilizó un muestreo aleatorio, es decir, todos los concurrentes a la playa (turistas, recreacionistas y habitantes del lugar) tuvieron la misma probabilidad de formar parte de la muestra. El tipo de muestreo fue de 'Bola de Nieve', al entrevistar a un grupo, se les pidió a las personas que indicasen a otros individuos que pudieran brindar información sobre el tema en cuestión (Martín-Crespo y Salamanca Castro, 2007).

Para determinar los espacios donde se encontraba el mayor número de visitantes durante el período seleccionado, se tomaron en cuenta relevamientos previos correspondientes a los Informes 2005/06 y 2006/07 de la DTCR. El mayor porcentaje de encuestas se realizaron los fines de semana donde el arribo de visitantes fue mayor. También se efectuaron los días miércoles con el fin de detectar personas que pernocten más de 3 noches. Las encuestas se llevaron a cabo en los sectores de: Punta Mingo; Calle $\mathrm{N}^{\circ}$ 5; Calle San Martín; Calle Espora; Calle Brown; Arbolito; Centro comercial y Campings (Fig. 2).

Todas las encuestas se realizaron en forma anónima. Se buscó caracterizar al visitante mediante las siguientes preguntas:

- Sexo y edad de los encuestados.

- Estado civil y grupo con el que concurrió.

- Nivel de educación y ocupación.

- Lugar de procedencia.

Luego se prosiguió con las preguntas sobre el destino en sí mismo y el uso de los servicios turísticos:

- Tiempo de estadía

- Frecuencia de visita.

- Servicios utilizados (transporte, alojamiento, gastronomía y recreación)

- Opinión sobre la calidad de los servicios de información y playa. 


\section{Figura 2 \\ ESQUEMA DEL PLANO DE PEHUÉN CO. LAS FLECHAS Y CÍRCULOS SEÑALAN LOS SECTORES DONDE LA DTCR REALIZÓ SUS ENCUESTAS}

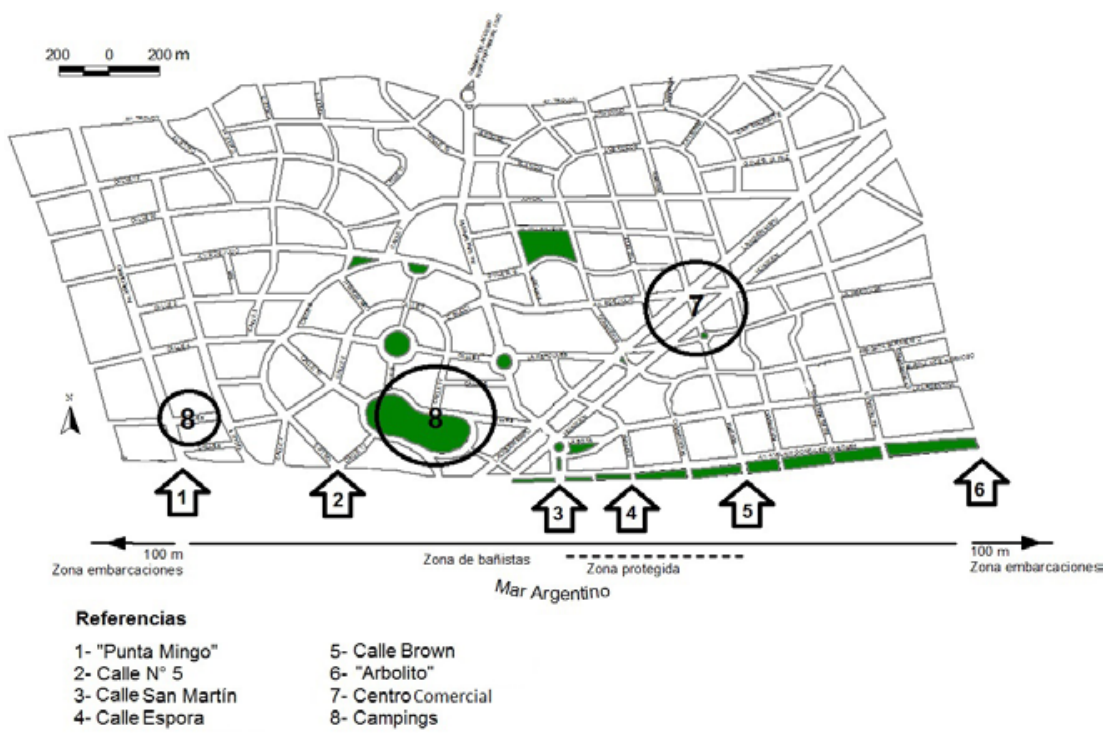

Fuente: elaboración propia.

- Aspectos generales (seguridad, condiciones de la playa, espacios verdes, limpieza, espacios públicos)

- Fortalezas y Debilidades del destino turístico.

Con el objetivo de saber si hay diversificación del turismo de sol y playa, se les consultó sobre «qué actividad realizan cuando no están en la playa», si «visitó otros sitios además de la playa» y si «en días o pronóstico de mal tiempo que actividades realizan».

Finalmente, se confeccionó un mapa de zonificación de áreas turísticas según su desarrollo. Esto fue con el fin de observar visualmente sobre el plano de la zona de estudio qué áreas y recursos están más desarrollados o no turísticamente. Para delimitar estas zonas se utilizó el análisis de la oferta de recursos turísticos disponibles y la demanda que afecta a esos recursos. De la oferta mencionada se tuvo en cuenta la disponibilidad y accesibilidad que tienen para el visitante, además de su situación actual, es decir, estado de conservación, competitividad con otros recursos y/o destinos, etc. La demanda se analizó mediante los resultados de las encuestas correspondientes a: lugares elegidos para la recreación; elementos determinantes de la elección de destino; fortalezas y debilidades que percibieron; etc. Posteriormente, se unieron los recursos con igual desarrollo turístico y se los relacionó con el grado de desarrollo de la demanda para cada recurso y el resultado se plasmó en el mapa de zonificación de áreas turísticas. Esto posibilitó la visualización de áreas potenciales de diversificación y la posterior generación de propuestas para su desarrollo. 


\section{RESULTADOS}

\subsection{Recursos turísticos}

Se identificaron los recursos turísticos que posee el balneario a escala local (Fig. 3) y regional (Fig. 4). Al analizarlos dentro del plano de la localidad balnearia se observó que los atractivos están concentrados en la cuadrícula SE, delimitada por las avenidas: 9 de Julio, San Martin, Rosales y González Martínez (Fig. 3). En este cuadrante encontramos:

1) Sala de interpretación «Florentino Ameguino»: Abierta sólo en temporada estival y vacaciones de invierno. Contiene abundante infografía sobre los yacimientos paleontológicos de la zona, exhibición de material fósil, maquetas, etc. Desde aquí se coordinan las excursiones guiadas a los yacimientos de huellas paleoicnológicas (Fig. 4).

2) Casa «Molino»: Es un edificio construido en forma de molino de viento en homenaje al libro Don Quijote de la Mancha. Actualmente cumple la función de vivienda familiar.

3) La Boya: Es uno de los edificios más antiguos. Tuvo múltiples usos: comedor, almacén, bar, carnicería, sodería, panadería, entre otros. Actualmente deshabitada.

4) Capilla: Se utiliza para celebraciones religiosas y durante el verano se organizan eventos de recreación para recaudar fondos.

5) Hotel «Cumelcan»: Concretado por un grupo de pobladores de la localidad en la temporada 1957/58. Fue el primer hotel del balneario. Hoy continúa funcionando bajo administración privada.

6) Casa «Barco»: Su propietario original fue el Sr. Luis Novelli que la diseñó, en 1954, según la forma del barco «Roma» donde él viajó desde Italia para radicarse en Argentina.

7) Feria de artesanos: Se congregan aproximadamente 30 expositores cada fin de semana de verano. Estos provienen principalmente de Pehuén Co, Punta Alta y Bahía Blanca, aunque también suele encontrarse artesanos del resto del país.

Los visitantes prefieren alojarse en zonas más cercanas al cuadrante SE, donde se encuentra la mayor cantidad de atractivos y comercios diurnos y nocturnos. Esto ocasiona que disminuya el valor turístico y económico en el resto de la localidad.

Entre los recursos de la zona circundante a la localidad balnearia (escala regional) se pueden observar diferentes yacimientos de huellas fósiles. En la playa, en las capas arcillo-limosas que se hallan cubiertas parcialmente por las altas mareas, se encuentran huellas fósiles de animales de 12.000 años de antigüedad. Estas fueron declaradas Reserva Natural Provincial Pehuén Co-Monte Hermoso en 2005. Es la primera y única de este tipo al poseer como objetivo el cuidado de los recursos paleontológicos, arqueológicos y geológicos. En la zona de estudio se divide en 2 áreas (Fig. 4): Area 1) «Las Rocas»: son acantilados de hasta $9 \mathrm{~m}$ de altura y una antigüedad de 3 a 5 millones de años. Incluye el yacimiento «Playa del Barco»: compuesto por afloramientos de rocas sedimentarias con material paleontológico de 16.000 años de antigüedad, este área se localiza en terrenos pertenecientes al Ejército Argentino y solo se puede acceder 


\section{Figura 3 \\ ESQUEMA DEL PLANO DE PEHUÉN CO Y SUS DIFERENTES ATRACTIVOS TURÍSTICOS}

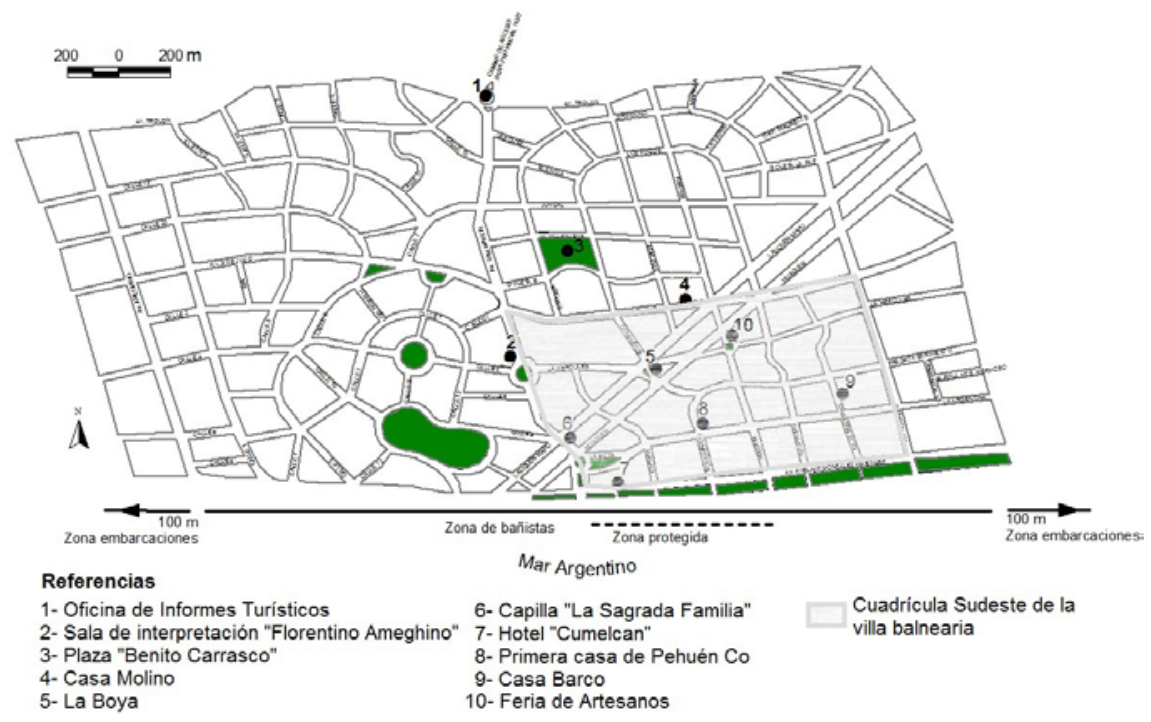

Fuente: elaboración propia.

\section{Figura 4 \\ RECURSOS TURÍSTICOS CERCANOS \\ A LA LOCALIDAD DE PEHUÉN CO}

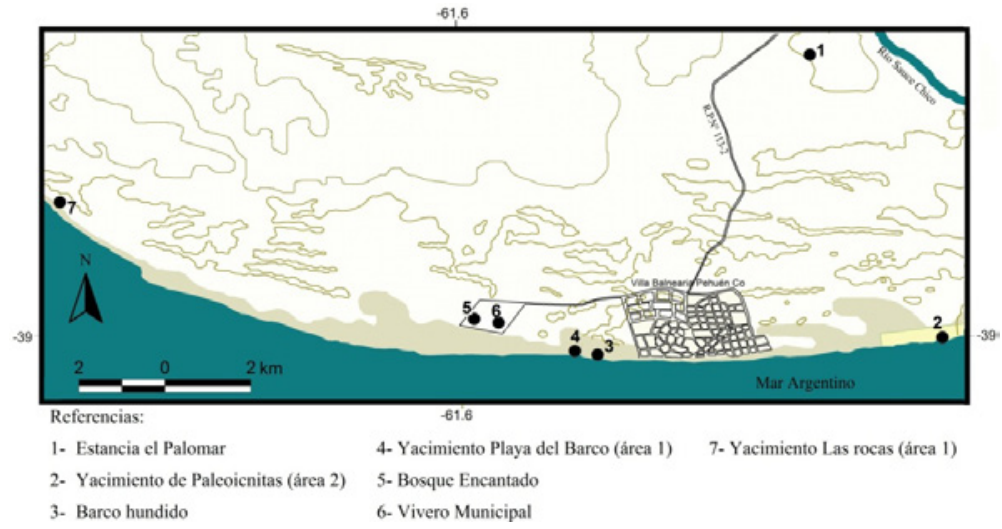

Fuente: elaboración propia. 
con permisos de paso y durante marea baja; Area 2) «Yacimiento de Paleoicnitas»: se encuentran huellas fósiles de mamíferos y aves con una antigüedad de 12.000 años.

Al Oeste de la villa se encuentra el denominado «Bosque Encantado» es un extenso bosque de 105 ha con especies arbóreas introducidas (pinos, eucaliptos, cipreses, etc.). En un principio funcionó como vivero municipal. En la actualidad funciona como camping municipal denominado «Bosque Encantado» (Fig. 4). También, al Oeste, sobre la playa, están los restos de un barco hundido en el Siglo XIX denominado «La Soberana» (Fig. 4) que solo se puede observar en marea baja. Este barco iba en lastre hacia Bahía Blanca, las piedras de ese lastre se utilizaron, entre otras cosas, para construir la capilla del balneario.

Colindantes al camino de acceso se encuentran varios cascos de estancias. En especial se destaca la estancia «El Palomar», distante a $8 \mathrm{~km}$ al Norte del balneario (Fig. 4). Esta posee un importante valor histórico y cultural en la región por haber sido el casco en el que se asentaba la familia propietaria de los terrenos donde se fundó Pehuén Co. Actualmente, continúa en manos de los sucesores de los fundadores de la localidad y se dedica principalmente a la ganadería. En el área circundante a la villa balnearia también se encuentran otras estancias distribuidas cerca del camino de acceso a Pehuén Co (R.P. N ${ }^{\circ}$ 112/3) que se dedican a la agricultura y ganadería, típicas actividades de la zona pampeana de Buenos Aires (Fig. 4). Estas actividades generan diferentes labores a lo largo de todo el año, como siembra, cosecha, engorde, cría, etc. Esto es una ventaja para poder lograr la diversificación del turismo de balneario, ya que son factibles de generar la participación de potenciales visitantes sin experiencia, por ejemplo en actividades de esquila de ovejas. Además, varias de estas estancias poseen casas con las dimensiones suficientes como para alojar por varios días a una o más familias que deseen realizar turismo o recreación en un campo típico de la pampa argentina.

\subsection{Perfil del visitante}

El resultado de las encuestas indica que las personas que eligieron Pehuén Co como destino fueron en su mayor parte recreacionistas, es decir, que solo concurrieron por el día, normalmente sábados o domingos. En cambio, el nivel de permanencia de los turistas fue alto, variando entre una y dos semanas. En su mayoría tenían entre 26 y 40 años con nivel educativo medio a alto. Generalmente eran grupos familiares (de 4 a 8 personas) o parejas que utilizaron vehículos particulares para llegar a su destino.

El principal lugar de procedencia correspondió a Bahía Blanca (ciudad distante a $80 \mathrm{~km}$ al Oeste con una población de 300.000 habitantes) (Fig. 1), aunque también se encontraron visitantes de otros municipios (Tornquist, Dorrego, La Plata, Buenos Aires) e incluso otras provincias (Mendoza y Neuquén). El lugar de procedencia marcó el rol del balneario como recurso provincial y/o nacional.

Las personas que en la última temporada (2010/11) concurrieron por primera vez al balneario argumentaron que fue por «el mejoramiento del camino de acceso a la villa». El camino mencionado se encontraba muy deteriorado y fue re-asfaltado en el invierno de 2010. La motivación para que los visitantes acudan a esta playa fue la búsqueda de «tranquilidad», «naturaleza»y «lejos del ruido y el cemento»según expresiones de los encuestados. 
Asimismo, al consultarles sobre las actividades realizadas en condiciones de mal tiempo, el $97 \%$ de las respuestas se dividió en: la permanencia en su alojamiento a la espera de que mejoren las condiciones del tiempo (24\%) y visitar localidades cercanas (73\%). Argumentaron principalmente la falta de actividades cuando no se podía visitar la playa por mal tiempo meteorológico. Esto demuestra la falta de diversificación de actividades en el lugar. Asimismo, esta diversificación debe realizarse bajo estricta planificación ya que el incremento de actividades recreativas podría alterar la mayor fortaleza del balneario (la tranquilidad y naturaleza).

En el período de estudio (2005 a 2011), se observó un cambio en el perfil del turista que visitó la playa de Pehuén Co. La cantidad de visitantes aumentó, esta modificación se produjo porque se redujo la cantidad de matrimonios con hijos y aumentó el número de parejas sin hijos que visitaron el balneario (Fig. 5). A su vez, se incrementó la cantidad de estudiantes universitarios, las personas procedentes de Bahía Blanca y las personas que visitaban por primera vez el balneario. Estos datos marcan un cambio en el tipo de demanda que visita la villa, pasando de ser un destino de familias a uno para personas más jóvenes sin hijos. Esta situación podría explicarse por un aumento de visitantes que accedieron en motocicleta, el cual es un vehículo particular para no más de dos personas esto refuerza el incremento de parejas sin hijos. El $26 \%$ de los encuestados fueron estudiantes universitarios, es decir con un nivel de educación y cultura medio a alto.

\section{Figura 5 \\ COMPOSICIÓN DEL GRUPO QUE VISITÓ PEHUÉN CO \\ EN LAS TEMPORADAS DE VERANO 2007 A 2011}

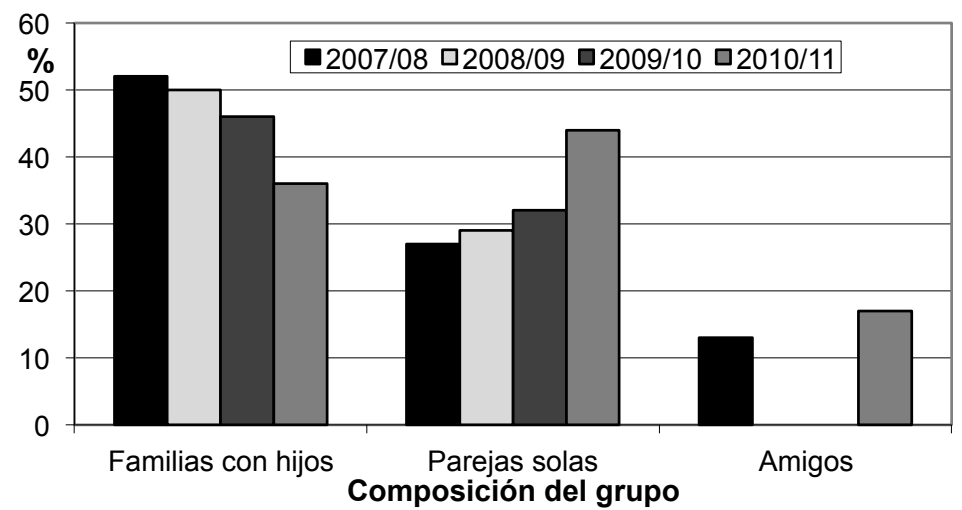

Fuente: elaboración propia.

En las encuestas de 2007/08 al consultar a los visitantes sobre si volverían a Pehuén Co la siguiente temporada estival, solo el $30 \%$ respondió afirmativamente. Asimismo, en 2010/11, el $87 \%$ de los encuestados manifestó que volverían el siguiente verano. Estos resultados muestran un cambio positivo en la demanda respecto de la visión del balneario como atractivo turístico. 
El conocimiento de las características de la demanda que concurre a Pehuén Co ayuda a diversificar su oferta turística. El nuevo segmento de visitantes se caracteriza por ser personas jóvenes, sin hijos y con un nivel de educación medio a alto. Por ello, es factible diversificar la oferta de sol y playa a otras actividades que apunten, por ejemplo, al turismo científico o de deportes. Especialmente durante los momentos del año en los que no es posible, por razones meteorológicas, disfrutar del sol y de la playa durante toda la estancia prevista por el turista (García Sánchez y Alburquerque García, 2003).

\subsection{Zonificación de las áreas turísticas según su desarrollo}

Es necesario el análisis de las características y evolución de la demanda turística de un destino para poder planificar la política turística centrada en la diversificación de productos turísticos (Molina Collado et al., 2007). Los visitantes mostraron una falta de interés en permanecer en Pehuén Co los días de mal tiempo o épocas de bajas temperaturas. Esto hace evidente la necesidad de una diversificación de la actividad bajo lineamientos que no afecten la mayor fortaleza competitiva de la localidad («la tranquilidad» según palabras de los encuestados). Por ello es necesario un MIC de toda la zona que se fundamente en estudios básicos e integrados que permitan identificar áreas potencialmente turísticas, teniendo en cuenta la disponibilidad de recursos, atractivos y las características de la demanda.

Se delimitaron tres tipos de áreas turísticas según su desarrollo: 1) Áreas turísticas bien desarrolladas, las que poseen un recurso fuerte y desarrollado y una demanda que conoce ese recurso y lo elige en su estadía, 2) Áreas turísticas parcialmente desarrolladas, coincide la demanda y la oferta pero alguna de las dos presenta alguna debilidad y 3) Áreas turísticas sin desarrollar, donde la oferta turística no existe o es muy débil y la demanda no es motivada hacia ese recurso (Fig. 6).

\section{Figura 6 \\ MAPA DE ZONIFICACIÓN TURÍSTICA DE PEHUÉN CO DE ACUERDO A SU GRADO DE DESARROLLO}

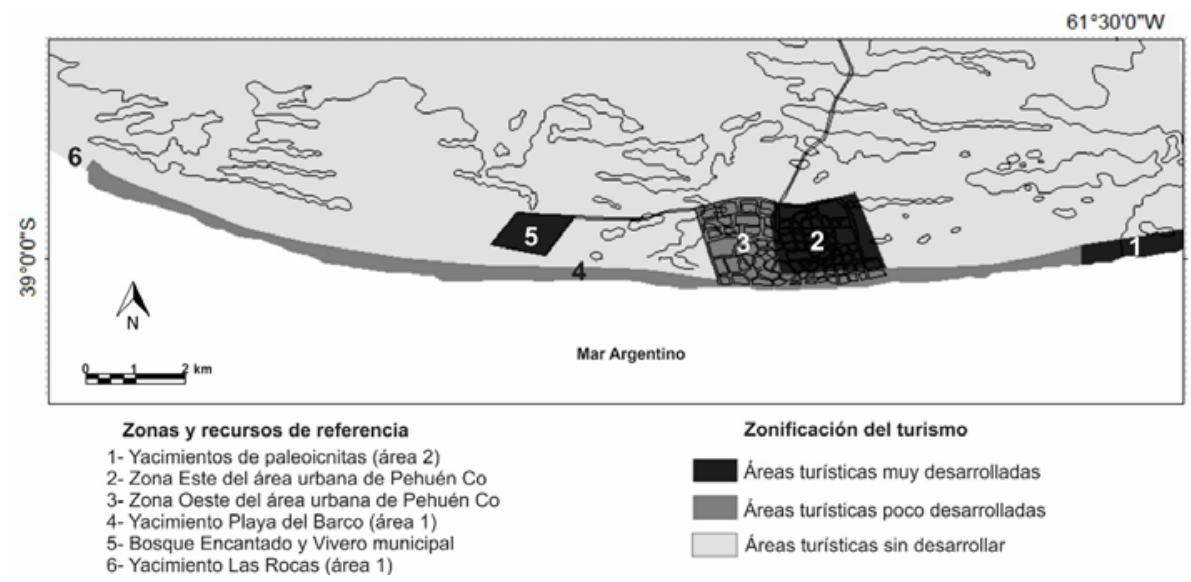

Fuente: elaboración propia. 
De esta forma, se identificó como áreas turísticas bien desarrolladas tres sectores: a) En la villa la zona al Este de la Av. San Martin, donde se localizaron la mayor cantidad de atractivos visitados por los turistas y recreacionistas (Casa Barco, Casa Molino, sala de interpretación, etc.) (Fig. 3); b) La zona del Bosque Encantado y Vivero Municipal (Fig. 4) y; c) El área 2 de la reserva paleontológica donde se realizaron visitas guiadas desde la villa (Fig. 4). En los sectores A y B, además de lo mencionado, se encuentra la mayor concentración de infraestructura necesaria para el turismo (hoteles, campings, restaurantes, comercios, etc.).

El área turística parcialmente desarrollada coincidió con el sector al Oeste de la Av. San Martin en la villa y la playa en sí misma. Esta zona de la villa presentó una debilidad en la cantidad y calidad de oferta de recursos turísticos. En tanto que en la playa, si bien la demanda coincidía en su elección y fue la motivación del viaje, el recurso en sí mostró algunas desventajas. Estas fueron la presencia de medusas, el estado de erosión activa que deja áreas de afloramientos de arena y conchilla cementada expuesta debajo del agua y la estacionalidad (en invierno la demanda no elige este producto). También la competencia directa que se genera con playas cercanas que ofrecen un producto de playa muy similar como, por ejemplo, Monte Hermoso (localizada $20 \mathrm{~km}$ al Este).

Por último, las áreas turísticas sin desarrollar fueron la zona del yacimiento «Las Rocas» (Fig. 4) y la zona agrícola-ganadera circundante a Pehuén Co. En el caso del yacimiento, si bien la demanda muestra una aceptación hacia este recurso, no puede acceder a él. Esto se debe a la extensa distancia a la que se encuentra, la inaccesibilidad en marea alta y por estar dentro de una propiedad del ejército argentino. En cuanto a las zonas de campos, si bien algunos podrían acondicionarse rápidamente para recibir visitantes, actualmente no presenta una oferta turística disponible (Fig. 6).

\section{PROPUESTAS PARA LA DIVERSIFICACIÓN TURÍSTICA Y CONCLUSIONES}

Pehuén Co cuenta con la mayor afluencia de turismo del partido de Coronel Rosales. Su economía se basa casi en su totalidad en el turismo de sol y playa. Debido a ello, es necesario reconocer otros espacios alternativos dentro de la región que complementen y diversifiquen la oferta turística actual. Este desarrollo es posible teniendo en cuenta los gustos, preferencias y motivaciones de la demanda. Por ello se analizó la demanda y se encontró que los grupos de mayor concurrencia al balneario son las familias con hijos, que por lo general optan por quedarse al menos una semana en el destino. Continúan las parejas de jóvenes que en su mayoría son recreacionistas y en ocasiones permanecen algunos días y, por último, los grupos de amigos que rara vez pernoctan en el destino. La motivación de la demanda hacia esta playa fue claramente la búsqueda de tranquilidad y naturaleza. Esta es la mayor fortaleza de este balneario respecto de otros cercanos con el mismo tipo de costa pero diferentes en cuanto a desarrollo urbano. Además, Pehuén Co cuenta con algunos productos alternativos a la playa, como atractivos puntuales dentro de la villa (casa Barco, casa Molino, etc.) y paseos, como el Bosque Encantado, el Vivero Municipal o el área 2 de la reserva paleontológica. Asimismo, requiere de mayor cantidad de oferta turística para los días de invierno o de mal tiempo meteorológico. 
Del análisis realizado se observó, principalmente, que hay zonas con potencial turístico. Por ello, se plantean propuestas con el fin de diversificar el turismo en las áreas con menos desarrollo de esta actividad (Tabla 2). Estas poseen atractivos reales (yacimiento Las Rocas) y potenciales (estancias cercanas) que pueden explotarse turísticamente y una demanda que puede hacer uso de esa oferta. Para comenzar con el uso de estos recursos sería necesario hacerlo en dos grandes líneas. Una correspondiente a la explotación del turismo científico (yacimientos fósiles) y otra al turismo rural. La primera debería hacerse en consenso con la localidad de Monte Hermoso (20 km al Este) dado que comparte algunas áreas de la reserva paleontológica. También con el Ejército Argentino $(10 \mathrm{~km}$ al Oeste) para considerar el trazado de un camino sobre su territorio para la visita del Área 1. Esto sumado a una mayor publicidad a nivel nacional de la existencia, características e importancia mundial de estos yacimientos puede generar una afluencia turística durante todo el año.

\section{Tabla 2}

\section{PROPUESTAS DE DIVERSIFICACIÓN TURÍSTICA PARA LAS DIFERENTES ÁREAS DE PEHUÉN CO}

\begin{tabular}{|c|c|}
\hline ÁREAS TURÍSTICAS & PROPUESTAS DE DIVERSIFICACIÓN TURÍSTICA \\
\hline Muy desarrolladas & $\begin{array}{l}\text { - Capacitación de los residentes para la recepción del turista } \\
\text { y la explotación sostenible de los recursos turísticos. } \\
\text { Promover el área a través de la publicidad nacional e inter- } \\
\text { nacional. }\end{array}$ \\
\hline Poco desarrolladas & $\begin{array}{l}\text { - Capacitación de los residentes para la recepción del turista } \\
\text { y la explotación sostenible de los recursos turísticos. } \\
\text { - Promover el área a través de la publicidad nacional e inter- } \\
\text { nacional. } \\
\text { Promocionar el Turismo científico en base a los recursos } \\
\text { paleontológicos. } \\
\text { - Generar un manejo integrado con Monte Hermoso y el } \\
\text { Ejército Argentino para el desarrollo del turismo paleon- } \\
\text { tológico. } \\
\text { - Aumentar la infraestructura para la recepción del turista. }\end{array}$ \\
\hline Sin desarrollar & 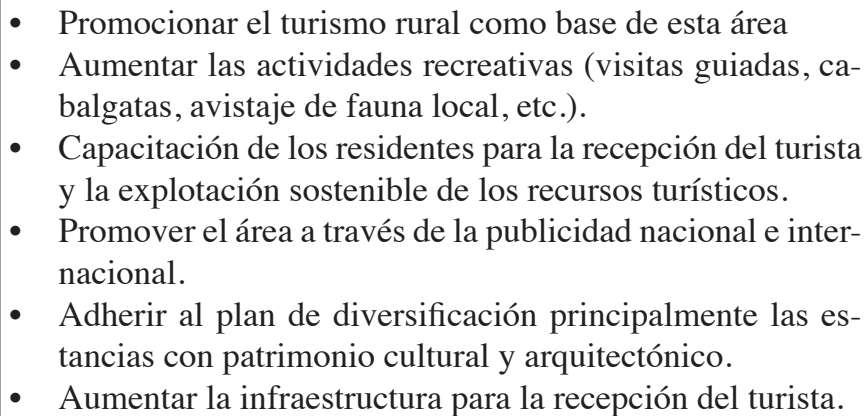 \\
\hline
\end{tabular}


El turismo rural es el de mayor potencialidad para la diversificación. Al aumentar el número de estancias que se adhieran al plan de diversificación mejor serían los resultados. Algunas de ellas poseen un patrimonio arquitectónico y cultural importante. Si se genera una puesta en valor de las mismas permitiría la diversificación del turismo y de las rentas. Esto ayudará a mantener este patrimonio histórico y optimizar los recursos existentes. Al igual que en el caso del turismo científico la publicidad a nivel regional y nacional es uno de los pasos más importantes para su desarrollo.

Una zonificación del área donde conjugan oferta y demanda turística es una de las bases para un MIC (Botero y Hurtado, 2009). Esta metodología se debe aplicar en toda la zona costera para poder lograr un manejo conjunto de los recursos, en especial una diversificación conjunta, competitiva y sustentable de los recursos naturales y turísticos. Sin olvidar que, para la elaboración del MIC, es necesaria una participación comprometida por parte de los habitantes de la localidad, sus tomadores de decisiones y de la comunidad científica.

\section{AGRADECIMIENTOS}

Los autores desean agradecer al Consejo Nacional de Investigaciones Científicas y Técnicas (CONICET), Universidad Nacional del Sur (UNS) y al proyecto COMET-LA (Gestión basada en la comunidad de los desafíos ambientales en América Latina) por el financiamiento del presente trabajo. También se agradece al Instituto Argentino de Oceanografía (IADO) y a la Dirección de Turismo del partido de Coronel Rosales.

\section{REFERENCIAS}

AMÉRIGO, M. (1993): Metodología de cuestionarios: Principios y aplicaciones. Departamento de Psicología Social. Universidad Complutense. Madrid. pp. 10.

AUSILI, G. (2005): Una propuesta de turismo cultural como oferta complementaria al atractivo natural del destino Pehuén Co. Tesis de licenciatura en Turismo, Departamento de Geografía y Turismo, Universidad Nacional del Sur, pp. 116.

BARTELUCCI, G. (1994): El turismo ecológico y las posibilidades de desarrollo en Pehuén Co. Tesis de licenciatura en geografía. Universidad Nacional del Sur, Bahía Blanca, pp. 48.

BOTERO, C. y HURTADO, Y. (2009): «Tourist Beach Sorts as a classification tool for Integrated Beach Management in Latin America». EUCC - Die Küsten Union Deutschland e.V.: International approaches of coastal research in theory and practice Coastline Reports, $\mathrm{n}^{\mathrm{O}}$ 13, pp. 133-142.

BUSTOS, M.L.y PICCOLO, M.C. (2011): «Desconfort térmico en verano e invierno para la localidad de Pehuén Co, Argentina». Nimbus. Revista de Meteorología, Climatología y Paisaje, Universidad de Almería. España, no 27-28, pp. 63-76.

BUSTOS, M.L., PICCOLO, M.C. y PERILLO, G.M.E. (2011): «Efectos geomorfológicos de fuertes vientos sobre playas. El caso de la playa de Pehuén Co, Argentina». Cuadernos de Investigación Geográfica, Universidad de La Rioja, España, vol. 37, n $^{\circ}$ 1, pp. 121-142. 
BUSTOS, M.L. (2012): Estudio integrado ambiental del balneario Pehuén Co. Tesis de doctorado (Inédita), Departamento de Geografía y Turismo, Universidad Nacional del Sur. pp. 231.

BUSTOS, M.L. y PICCOLO, M.C. (2012): «Esquema de información climática para turismo aplicado al balneario Pehuén Co, Argentina». Territoris, Universidad de Islas Baleares. España, no 8, pp. 29-40.

CALO, J., FERNÁNDEZ, E., MARCOS, A y ALDACOUR, H. (1998): «Análisis preliminar del balance sedimentario de la playa de Pehuén Co, provincia de Buenos Aires, Argentina». GEOACTA, no 23, pp. 1-12.

CENGIZ, T., AKBULAK, C., CALISKAN, V. y KELKIT, A. (2008): «Climate Comfortable for Tourism: A Case Study of Canakkale». BALWOIS 2008, Macedonia, pp. 1-9.

CHANG Y. C., HONG, F. W. y LEE, M. T. (2008): «A system dynamic based DSS for sustainable coral reef management in Kenting coastal zone, Taiwan». Ecological Modeling, $\mathrm{n}^{\mathrm{o}} 211$, pp. 153-168.

COMISIÓN EUROPEA (2001): La Unión Europea apuesta por las zonas costeras. Luxemburgo: Oficina de Publicaciones Oficiales de las Comunidades Europeas, 29 pp.

CUTIDIANO, R. (2010): Evaluación del potencial turístico de la oferta y demanda: aplicación a la localidad de Pehuén Co. Tesis de licenciatura en Turismo, Departamento de Geografía y Turismo, Universidad Nacional del Sur, pp. 99.

DADON, J. R., CHIAPPINI, G. y RODRÍGUEZ, M.C. (2002): «Impactos ambientales del turismo costero en la provincia de Buenos Aires». Gerencia Ambiental, vol. 9, $\mathrm{n}^{\mathrm{o}}$ 88 , pp. 552-560.

D’AMATO, S. (2011): Capacidad de carga turística en el Área 2 de la Reserva Natural Provincial Pehuén Co - Monte Hermoso: Propuesta de un sendero educativo interpretativo. Tesis de licenciatura en Turismo, Departamento de Geografía y Turismo, Universidad Nacional del Sur, pp. 135.

DEL POZO, O. (2001): El proceso de urbanización y degradación ambiental del ecosistema costero: Municipio de Monte Hermoso. Provincia de Buenos Aires. Tesis de Maestría en gestión ambiental del desarrollo urbano, Universidad Nacional del Sur, Bahía Blanca, pp. 249.

DEL POZO O., BAZÁN S. y ROSELL M. (2000): El turismo, la apropiación de los recursos costeros y la preservación del ambiente. Un estudio de caso en el suroeste bonaerense. Turismo: Desarrollo local y regional. Ediuns, Bahía Blanca, pp. 145-160.

DOUVERE, F. (2008): «The importance of marine spatial planning in advancing ecosystem-based sea use management». Marine Policy, $\mathrm{n}^{\circ}$ 32, pp. 762-771.

GARCÍA SÁNCHEZ, A. y ALBURQUERQUE GARCÍA, F. (2003): «El turismo cultural y el de sol y playa ¿Sustitutivos o complementarios?», Cuadernos de Turismo, $\mathrm{n}^{\circ} 11$, pp. 97-105.

GESAMP (Grupo Mixto de Expertos Sobre los Aspectos Científicos de la Protección del Medio Marino) (1999): «La contribución de la ciencia al manejo costero integrado». Inf. Estud. GESAMP, pp. 61-65.

GIUliETTI, M. (2010): El turismo científico en Pehuén Co. Propuestas para la implementación de un nuevo producto turístico. Tesis de licenciatura en Turismo, Departamento de Geografía y Turismo, Universidad Nacional del Sur, pp. 84. 
GÓMEZ MARTÍN, B. (1999): «La relación clima-turismo: consideraciones básicas en los fundamentos teóricos y prácticos». Investigaciones Geográficas, $\mathrm{n}^{\circ}$ 21, pp. 21-34.

COI (COMISIÓN OCEANOGRÁFICA INTERGUBERNAMENTAL) (2004): $37^{\circ}$ reunión del Consejo Ejecutivo, París, 23-29 de junio de 2004, EC-37/2 Anexo 8, pp. 7.

LESLIE, H. M. y MCLEOD, K.L. (2007): «Confronting the challenges of implementing marine ecosystem-based Management». Review Front Ecol Environ, vol. 5, $\mathrm{n}^{\circ}$ 10, pp. 540-548.

MOLINA COLLADO, A., CONSUEGRA NAVARRO, D., TALAYA, A. y DÍAZ SÁNCHEZ, E. (2007): «Segmentación de la demanda turística: un análisis aplicado a un destino de turismo cultural». Revista de Análisis Turístico, no 4, pp. 36-48.

MONTANER MONTEJANO, J. (1996): Psicosociología del turismo. Síntesis, España, pp. 240.

MORENO CASTILLO, I. (2007): Manejo integral Costero. Por una costa más ecológica, productiva y sostenible. Universitat de les Illes Balears, Vol. 3, pp. 217.

OMT (ORGANIZACIÓN MUNDIAL DE TURISMO) (1998): Introducción al turismo. OMT, pp. 392.

OMT (ORGANIZACIÓN MUNDIAL DE TURISMO) (2008): International Recommendations for Tourism Statistics 2008. United Nations Publication, Series M, 83 (1), pp. 55.

OMT (ORGANIZACIÓN MUNDIAL DE TURISMO) (2009): Barómetro OMT del turismo mundial. United Nations Publication, vol. 7 (1), pp. 60.

PERILLO, G.M.E. (1992): «Control y cuidado de nuestras costas». La Nueva Provincia 4 partes, días 4, 11, 18 y 28 de febrero de 1992, pp. 7.

SÁNCHEZ, M. (2010): Incidencia de la repavimentación del camino de acceso a Pehuén Co en un nuevo perfil turístico para el balneario. Tesis de licenciatura en Turismo, Departamento de Geografía y Turismo, Universidad Nacional del Sur, pp. 49.

SCOTT, D. y MCBOYLE, G. (2001): «Using a 〈tourism climate index» to examine the implications of climate change for climate as a natural resource for tourism». En Matzarakis, A. y de Freitas, C. (Eds.), Proceedings of the First International Workshop on Climate, Tourism and Recreation. International Society of Biometeorology, Commission 5, Halkidi, Greece, pp. 69-98.

SUÁREZ DE VIVERO J.L. Y RODRÍGUEZ MATEOS J.C. (2005): «Coastal crisis: the failure of coastal management in the spanish mediterranean region». Coastal Management, $\mathrm{n}^{\mathrm{o}} 33$, pp. 197-214.

WONG, P. (1998): «Coastal tourism development in Southeast Asia: relevance and lessons for coastal zone management». Ocean \& Coastal Management, $n^{\circ} 38$, pp. 89-109. 
